\title{
INTRODUCTION
}

\section{Targeting TRP Channels for Pain Relief: TRPV1 and Beyond}

\author{
Man-Kyo Chung*
}

\author{
Department of Neural and Pain Sciences, School of Dentistry, Program in Neuroscience, University of Maryland, 650 \\ W. Baltimore Street, Baltimore, MD 21201, USA
}

Management of chronic and pathological pain without incurring systemic side effects is a major medical challenge. Currently available drugs, such as non-steroidal antiinflammatory drugs or opioid agonists, are efficacious through peripheral and central mechanisms. However, various complications and development of tolerance are serious problems. Other classes of drugs, such as antidepressants and anti-convulsants, are often used for multiple pain syndromes. However, the efficacy of these drugs is commonly unsatisfactory, and their mechanism of action is not clear. For establishing novel, selective anti-hyperalgesic therapeutic approaches, targeted inhibition of pain-specific pathways or molecules would be ideal, and these approaches suggest straightforward strategies. A new era of exploring such "straightforward" approaches was opened with regard to peripheral nociceptors by the identification of the vanilloid receptor-1 (VR-1), which was designated transient receptor potential channel vanilloid subtype 1 (TRPV1). TRPV1 is a receptor for capsaicin, proton, and noxious heat. Capsaicin has long been known to be a natural compound capable of evoking an intense burning sensation and pain in human and experimental animals. It has been hypothesized that specific manipulation of TRPV1 may selectively relieve pain under injury or inflammatory conditions. Interfering with TRPV1 has been a central focus of these efforts during the 15 years following the cloning of TRPV1. Numerous pharmacological compounds have been developed targeting TRPV1. The characteristics and roles of TRPV1 have been rigorously studied using multiple approaches ranging from biophysical characterization to clinical trials in human subjects. Meanwhile, other members of the TRP channel family in addition to TRPV1 have been suggested to be also involved in nociception under pathophysiological conditions. These studies have identified targets in addition to TRPV1 as potential candidates for selective anti-hyperalgesic treatment free from complications.

In this special issue of The Open Pain Journal, current knowledge regarding the roles of various TRP channels in pain is reviewed. Multiple scientists in academia and the pharmaceutical industry took part in this exciting project, and have shared their opinions regarding the prospects for relieving pain through targeting TRP channels.

*Address correspondence to this author at the Department of Neural and Pain Sciences, School of Dentistry, Program in Neuroscience, University of Maryland, 650 W. Baltimore Street, Baltimore, MD 21201, USA;

Tel: 410-706-4452; Fax: 410-706-0865; E-mail: MChung@umaryland.edu
In the opening chapter, "Changes in TRP channels expression in painful conditions," Bishnoi and Premkumar summarize the involvement of various members of the TRP channel family in nociception. The five following chapters are devoted to a discussion of the role of various TRP channels in specific pathological conditions. Blackshaw and colleagues review the evidence for the roles of "TRP channels in visceral pain." They discuss the contribution of TRP channels, especially TRPV1, TRPV4, TRPA1, and TRPM8, to pain signaling in visceral systems in various visceral pathological models. The roles of "TRP channels in dental pain" are reviewed by Chung and Oh. The expression of various TRP channels in pulpal afferents and odontoblasts is summarized, and their potential contribution to dental pain by thermal and mechanical stimuli is discussed. In "The role of TRP channels in migraine," Oxford and Hurley review the potential roles of TRP channels in a trigeminovascular system, their involvement in migraine attack, and recent clinical trials. Fernandes et al. discuss "TRP receptors in arthritis, gaining knowledge for translation from experimental models". They review current knowledge pertaining to the therapeutic potential of TRP channels, primarily TRPV1 and TRPA1, for treating pain in osteoarthritis and other arthritic conditions. The roles of "Transient Receptor Potential channels in chemotherapyinduced neuropathy" are discussed by Nassini et al. In this chapter, the current understanding of the involvement of TRPV1, TRPV4, TRPM8, and TRPA1 in chemotherapyinduced neuropathy is highlighted.

The next three chapters analyze the status of therapeutic approaches targeting TRPV1 using specific agonists and antagonists. In effects of topical capsaicin on cutaneous innervation: Implications for pain management Bley reviews the potential mechanisms of topical application of high concentrations of capsaicin to skin, and suggests pharmacokinetic and pharmacodynamic considerations of this therapy. Iadarola and colleagues discuss how sitespecific administration of resiniferatoxin, an ultrapotent capsaicin analog, can overcome the limitations of topical capsaicin therapy, and they give an insight into the clinical trials using resiniferatoxin for permanent pain relief in cancer patients with intractable pain. Trevisani and Gatti discuss "TRPV1 antagonists as analgesic agents." The authors review preclinical and clinical studies addressing the application of small molecule TRPV1 antagonists as anti-hyperalgesic therapies, and update current status of clinical trials. 
The following three chapters provide detailed discussion and information regarding targeting three TRP channels, TRPA1, TRPV3, and TRPM8, for anti-hyperalgesic therapy. The "Roles of TRPA1 in pain pathophysiology and implications for the development of a new class of analgesic drugs" are discussed by Radresa et al. The authors provide a comprehensive review of the properties of TRPA1 and the roles of TRPA1 in multiple pathological pain conditions; the current status of clinical trials using TRPA1 antagonists is updated. Huang and Chung discuss "Targeting TRPV3 for the development of novel analgesics." They summarize current knowledge of physiology and pharmacology of TRPV3, its contribution to thermosensation and nociception, as well as to other skin pathophysiology. Fernández-Peña and Viana review "Targeting TRPM8 for Pain Relief." In this chapter, they discuss not only the major role of TRPM8 in cold pain, but also in a variety of conditions, such as dry eye disease, airway irritation, tooth hypersensitivity, migraine, and neuropathic pain. In addition to nociception, current knowledge regarding the contribution of TRP channels to itch sensation is discussed by Biro and coauthors.

As suggested in this collection of reviews, there is no question but that various TRP channels are fascinating candidates for suppressing pain under multiple pathological conditions. The preclinical and clinical studies using TRPV1 antagonists, however, strongly suggest that selective suppression of pathological pain without side effects is difficult to achieve through a straightforward approach, e.g., by suppressing the entire function of the given ion channel. This lesson should be seriously considered with respect to other TRP channels which are targeted in anti-hyperalgesic approaches. Most TRP channels involved in nociception also contribute to non-pain functions; therefore, the multifunctional nature of TRP channels requires more effort and creativity by researchers seeking to translate bench research into clinically applicable therapeutics. Further studies will be needed in order to develop compounds suitable for targeting pathology-specific changes in TRP channels that contribute to hyperalgesia. Differential regulation and functions of a given TRP channel in different locations in pain pathways or different tissues needs to be further investigated to establish site- or tissue-specific targeting approaches. It will be essential to have more information regarding the structural basis of activation, sensitization, and desensitization of TRP channels for developing therapeutics that target a particular sub-receptor region. The reviews in this special issue should be reliable checkpoints as we continue the journey toward developing novel strategies for relieving pathological pain.

(C) Man-Kyo Chung; Licensee Bentham Open.

This is an open access article licensed under the terms of the Creative Commons Attribution Non-Commercial License (http:/creativecommons.org/licenses/by-nc/3.0/) which permits unrestricted, non-commercial use, distribution and reproduction in any medium, provided the work is properly cited. 